\title{
Some Series and Mathematic Constants Arising in Radioactive Decay
}

\author{
Xun Zhou ${ }^{1,2}$ \\ ${ }^{1}$ School of Water Resources and Environment, China University of Geosciences (Beijing), Beijing, China \\ ${ }^{2}$ MOE Key Laboratory of Groundwater Circulation and Environmental Evolution, China University of Geosciences \\ (Beijing), Beijing, China \\ Correspondence: Xun Zhou, School of Water Resources and Environment, China University of Geosciences (Beijing), \\ Xueyuan Road 29, Beijing 100083, P. R. China. E-mail: zhouxun@ @ugb.edu.cn
}

Received: October 2, 2019

Accepted: October 23, 2019 Online Published: October 28, 2019

doi:10.5539/jmr.v11n6p14

URL: https://doi.org/10.5539/jmr.v11n6p14

\begin{abstract}
In this paper we show the construction of 32 infinite series based on the law of decay of radioactive isotopes, which indicates that a radioactive parent isotope is reduced by $1 / 2$ and $1 / e$ of its initial value during each half-life and mean life, respectively. We found that the ratios among the values of the radioactive parent isotope and the radiogenic daughter isotope for each half-life's and mean life's decay can be used to construct 16 half-life related (or 2-related) and 16 mean life related (or $e$-related) infinite series. There are 8 divergent series, 4 previously known convergent series and 2 series converging to the Erdös-Borwein constant. The remaining 18 series are found to converge to 18 mathematical constants and the divergent and alternating mean life related series leads to another 2 mathematical constants. A few interesting mathematical relations exist among these convergent series and 5 sequences are also attained from the convergent half-life related series.
\end{abstract}

Keywords: infinite series, mathematical constant, radioactive decay, radioactive isotope, sequence

\section{Introduction}

Mathematical constants were found or arise in a variety of ways. Many mathematical constants are associated with number theory, analytic inequalities, enumerating discrete structures, functional iteration, complex analysis and geometry, and so on (Finch, 2003; Choi, 2012). $\sqrt{2}=1.414 \cdots$, which is sometime called the Pythagoras' constant, is associated with geometry when the length of the diagonal of a unit square was determined (Finch, 2003). Quite a few mathematical constants are the sums of convergent infinite series or have series representations (Alzer, Karayannakis, \& Srivastava, 2006). The Apéry's constant, for instance, is the sum of the convergent infinite series: $1+1 / 2^{3}+1 / 3^{3}+1 / 4^{3}+1 / 5^{3}+\cdots=1.202056 \cdots$ (M. Chen, \& S. Chen, 2016; R. Chen, 2011). A divergent infinite series may also lead to mathematical constants if its partial sum is bounded. The Marvin Ray Burns' (MRB) constant is the upper bounded value of the partial sum of the divergent and alternating infinite series: $-1^{1 / 1}+2^{1 / 2}-3^{1 / 3}+4^{1 / 4}-5^{1 / 5}+6^{1 / 6} \cdots=0.187859 \cdots$ (M. Chen, \& S. Chen, 2016). Thus, construction of new infinite series has the possibility of leading to new mathematical constants.

\section{Half-Life Related (or 2-Related) Infinite Series}

Radioactive isotopes (e. g., ${ }^{14} \mathrm{C}$ ) are used to determine the age of some geological materials, such as travertines or tufas depositing from hot springs or ordinary-temperature springs. Radioactive parent isotopes spontaneously radiate rays and decays continually, and radiogenic daughter isotopes grow simultaneously (Faure, 1979; Lutgens \& Tarbuck, 2014; Turcotte \& Schubert, 2002). During a single-component decay, the value of atom of the radioactive parent isotope decays in a negative exponential function, and the value of atom of the radiogenic daughter isotope grows, respectively, as follows (Faure, 1979; Turcotte \& Schubert, 2002):

$$
P(t)=P(0) e^{-\lambda t},
$$

and

$$
D(t)=P(0)\left(1-e^{-\lambda t}\right),
$$

where $P(0)$ is the initial value of atom of the radioactive parent isotope, $P(t)$ and $D(t)$ are the values of atoms of the radioactive parent isotope and the radiogenic daughter isotope at time $t$, respectively, and $\lambda$ is the decay constant of the radioactive parent isotope. The term, half-life $\left(T_{1 / 2}\right)$, which is defined to be the time required for one-half of the atoms of the parent isotope present at the initial moment to decay, is used to describe the velocity of decay, and $T_{1 / 2}=\ln 2 / \lambda$. 
Therefore, Equations (1) and (2) can be rewritten using half-life as follows:

$$
P(i)=P(0) e^{-\lambda T_{1 / 2}(i)},
$$

and

$$
D(i)=P(0)\left(1-e^{-\lambda T_{1 / 2}(i)}\right),
$$

where $i$ is the number of half-life of decay of the radioactive parent isotope, $P(i)$ and $D(i)$ are the values of atoms of the radioactive parent isotope and the radiogenic daughter isotope after $i$ th half-life's decay, respectively. Both the decay of $P(i)$ and the increase of $D(i)$ with $i$ when $P(0)$ is assumed to be 120 are shown in Figure 1 . It is easy to see that $P(i)=D(i)=60$ when $i=1$.

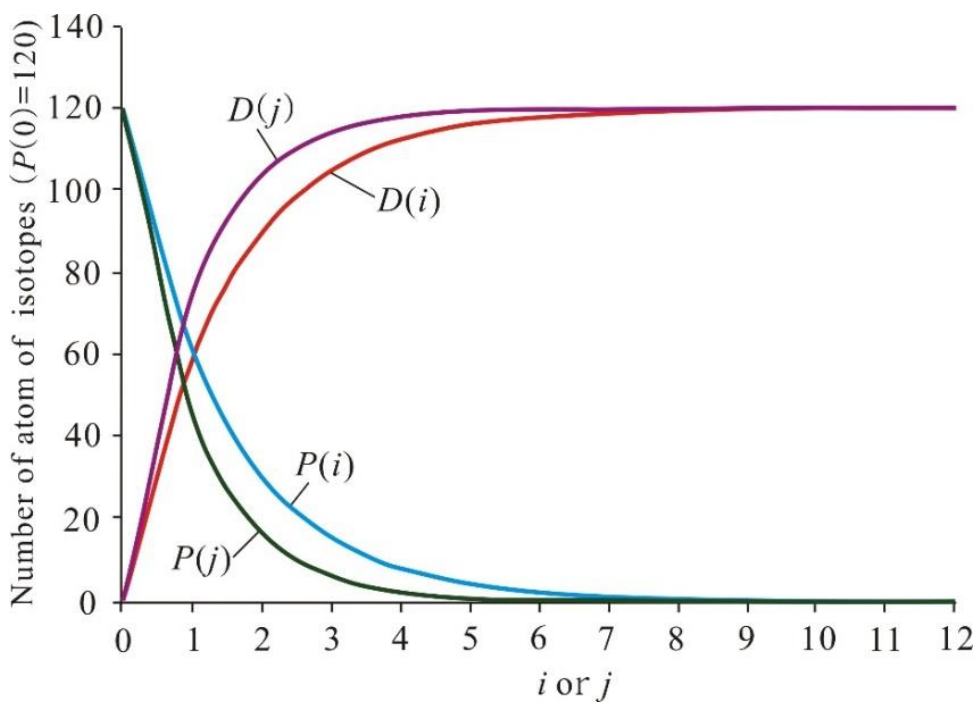

Figure 1. Changes of $P(i)$ and $D(i)$ with $i$ and $P(j)$ and $D(j)$ with $j$, and curves of $P(i)$ and $D(i)$ are modified after Faure (1977)

Obviously, the radioactive parent isotope is reduced by a factor of $1 / 2$ during each half-life (Faure, 1977). The remaining values of the radioactive parent isotope and the accumulating values of the radiogenic daughter isotope from 1 to 10 half-life's decay when $P(0)=120$ are listed in Table 1 . Also listed in Table 1 are the ratios of $P(i) / P(0), D(i) / P(0), P(i) / D(i)$ and $(P(i) / P(0)) \cdot(P(i) / D(i))$ at $i$ th half-life, $T_{1 / 2}(i), i=1,2,3, \cdots, 10, \cdots$.

Table 1. Decrease in the radioactive parent isotope and increase in the radiogenic daughter isotope during each half-life in a single-component decay

\begin{tabular}{cccccccc}
\hline $\mathrm{C} 1$ & $\mathrm{C} 2$ & $\mathrm{C} 3$ & $\mathrm{C} 4$ & $\mathrm{C} 5$ & $\mathrm{C} 6$ & $\mathrm{C} 7$ & $\mathrm{C} 8$ \\
\hline$i$ & $T_{1 / 2}(i)$ & $P(i)$ & $D(i)$ & $\frac{P(i)}{P(0)}$ & $\frac{D(i)}{P(0)}$ & $\frac{P(i)}{D(i)}$ & $\frac{P(i)}{P(0)} \cdot \frac{P(i)}{D(i)}$ \\
\hline 1 & $\ln 2 / \lambda$ & 60 & 60 & $1 / 2$ & $1 / 2$ & 1 & $1 / 2$ \\
2 & $2 \ln 2 / \lambda$ & 30 & 90 & $1 / 4$ & $3 / 4$ & $1 / 3$ & $1 / 12$ \\
3 & $3 \ln 2 / \lambda$ & 15 & 105 & $1 / 8$ & $7 / 8$ & $1 / 7$ & $1 / 56$ \\
4 & $4 \ln 2 / \lambda$ & 7.5 & 112.5 & $1 / 16$ & $15 / 16$ & $1 / 15$ & $1 / 240$ \\
5 & $5 \ln 2 / \lambda$ & 3.75 & 116.25 & $1 / 32$ & $31 / 32$ & $1 / 31$ & $1 / 992$ \\
6 & $6 \ln 2 / \lambda$ & 1.875 & 118.125 & $1 / 64$ & $63 / 64$ & $1 / 63$ & $1 / 4032$ \\
7 & $7 \ln 2 / \lambda$ & 0.9375 & 119.0625 & $1 / 128$ & $127 / 128$ & $1 / 127$ & $1 / 16256$ \\
8 & $8 \ln 2 / \lambda$ & 0.46875 & 119.53125 & $1 / 256$ & $255 / 256$ & $1 / 255$ & $1 / 65280$ \\
9 & $9 \ln 2 / \lambda$ & 0.234375 & 119.765625 & $1 / 512$ & $511 / 512$ & $1 / 511$ & $1 / 261632$ \\
10 & $10 \ln 2 / \lambda$ & 0.1171875 & 119.8828125 & $1 / 1024$ & $1023 / 1024$ & $1 / 1023$ & $1 / 1047552$
\end{tabular}

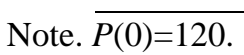

When 1 is added into column C5 in Table 1, from the fractions we can construct the previously known infinite geometric series, including the following positive term series XA, alternating series XB, odd term series XC and even term series $\mathrm{XD}$, and give their sums: 


$$
\begin{gathered}
\mathrm{XA}=1+\frac{1}{2}+\frac{1}{4}+\frac{1}{8}+\frac{1}{16}+\frac{1}{32}+\frac{1}{64}+\frac{1}{128}+\frac{1}{256}+\frac{1}{512}+\frac{1}{1024}+\cdots=\sum_{i=0}^{\infty} \frac{1}{2^{i}}=2 \\
\mathrm{XB}=1-\frac{1}{2}+\frac{1}{4}-\frac{1}{8}+\frac{1}{16}-\frac{1}{32}+\frac{1}{64}-\frac{1}{128}+\frac{1}{256}-\frac{1}{512}+\frac{1}{1024}-\cdots=\sum_{i=0}^{\infty}(-1)^{i} \frac{1}{2^{i}}=\frac{2}{3} \\
\mathrm{XC}=1+\frac{1}{4}+\frac{1}{16}+\frac{1}{64}+\frac{1}{256}+\frac{1}{1024}+\cdots=\sum_{i=0}^{\infty} \frac{1}{2^{2 i}}=\frac{4}{3} \\
\mathrm{XD}=\frac{1}{2}+\frac{1}{8}+\frac{1}{32}+\frac{1}{128}+\frac{1}{512}+\cdots=\sum_{i=1}^{\infty} \frac{1}{2^{2 i-1}}=\frac{1}{2} \sum_{i=0}^{\infty} \frac{1}{2^{2 i}}=\frac{2}{3} .
\end{gathered}
$$

It is easy to find that $\mathrm{XB}=\mathrm{XA} / 3, \mathrm{XC}=2 \mathrm{XA} / 3, \mathrm{XC}=2 \mathrm{XB}, \mathrm{XC}=\mathrm{XA}-\mathrm{XB}, \mathrm{XC}=(\mathrm{XA}+\mathrm{XB}) / 2, \mathrm{XC}=\mathrm{XA} \cdot \mathrm{XB}, \mathrm{XD}=\mathrm{XA} / 3$, $\mathrm{XD}=\mathrm{XB}, \mathrm{XD}=\mathrm{XC} / 2, \mathrm{XD}=\mathrm{XA}-2 \mathrm{XB}, \mathrm{XD}=\mathrm{XA}-\mathrm{XC}$ and $\mathrm{XD}=\mathrm{XC}-\mathrm{XB}$.

When 1 is added into column C6 in Table 1, the following positive term series XE, alternating series XF, odd term series $\mathrm{XG}$ and even term series XH can be constructed:

$$
\begin{gathered}
\mathrm{XE}=1+\frac{1}{2}+\frac{3}{4}+\frac{7}{8}+\frac{15}{16}+\frac{31}{32}+\frac{63}{64}+\frac{127}{128}+\frac{255}{256}+\frac{511}{512}+\frac{1023}{1024}+\cdots=1+\sum_{i=1}^{\infty} \frac{2^{i}-1}{2^{i}}=\infty ; \\
\mathrm{XF}=1-\frac{1}{2}+\frac{3}{4}-\frac{7}{8}+\frac{15}{16}-\frac{31}{32}+\frac{63}{64}-\frac{127}{128}+\frac{255}{256}-\frac{511}{512}+\frac{1023}{1024}-\cdots=1+\sum_{i=1}^{\infty}(-1)^{i} \frac{2^{i}-1}{2^{i}}=\left\{\begin{array}{c}
\frac{4}{3} \\
\frac{1}{3}
\end{array}\right. \\
\mathrm{XG}=1+\frac{3}{4}+\frac{15}{16}+\frac{63}{64}+\frac{255}{256}+\frac{1023}{1024}+\cdots=1+\sum_{i=1}^{\infty} \frac{2^{2 i}-1}{2^{2 i}}=\infty ; \\
\mathrm{XH}=\frac{1}{2}+\frac{7}{8}+\frac{31}{32}+\frac{127}{128}+\frac{511}{512}+\cdots=\sum_{i=1}^{\infty} \frac{2^{2 i-1}-1}{2^{2 i-1}}=\infty .
\end{gathered}
$$

Series XE, XG and XH are obviously divergent. Series XF also diverges since when $i \rightarrow \infty$, its $i$ th term is not equal to 0 and the partial sum approaches alternatively to the upper bounded value $X F U=4 / 3$ and the lower bounded value $X F L=1 / 3$. It is easy to obtain: $\mathrm{XFU}-\mathrm{XFL}=1$.

From column C7 in Table 1, i.e., the ratios of the remaining numbers of the radioactive parent isotope to the accumulating numbers of the radiogenic daughter isotope for each half-life (Lutgens \& Tarbuck, 2014), we can write the following positive term series XI, alternating series XJ, odd term series XK and even term series XL:

$$
\begin{gathered}
\mathrm{XI}=1+\frac{1}{3}+\frac{1}{7}+\frac{1}{15}+\frac{1}{31}+\frac{1}{63}+\frac{1}{127}+\frac{1}{255}+\frac{1}{511}+\frac{1}{1023}+\cdots=\sum_{i=1}^{\infty} \frac{1}{2^{i}-1}=1.606695 \cdots ; \\
\mathrm{XJ}=1-\frac{1}{3}+\frac{1}{7}-\frac{1}{15}+\frac{1}{31}-\frac{1}{63}+\frac{1}{127}-\frac{1}{255}+\frac{1}{511}-\frac{1}{1023}+\cdots=\sum_{i=1}^{\infty}(-1)^{i+1} \frac{1}{2^{i}-1}=0.764499 \cdots ; \\
\mathrm{XK}=1+\frac{1}{7}+\frac{1}{31}+\frac{1}{127}+\frac{1}{511}+\frac{1}{2047}+\cdots=\sum_{i=1}^{\infty} \frac{1}{2^{2 i-1}-1}=1.185597 \cdots ; \\
\mathrm{XL}=\frac{1}{3}+\frac{1}{15}+\frac{1}{63}+\frac{1}{255}+\frac{1}{1023}+\cdots=\sum_{i=1}^{\infty} \frac{1}{2^{2 i}-1}=0.421097 \cdots .
\end{gathered}
$$

The sum of series XI is exactly the sum of the reciprocals of the Mersenne's numbers. XI=1.606695 $\ldots$ is known as the Erdös-Borwein constant, whose irrationality was proved by Erdös (1948) and Borwein (1992). XI is also known as one of digital search tree constants (Finch, 2003). It is easy to get: $\mathrm{XI}=\mathrm{XK}+\mathrm{XL}$ and $\mathrm{XJ}=\mathrm{XK}-\mathrm{XL}$.

When 1 is added into column C8 in Table 1, the following positive term series XM, alternating series XN, odd term series $\mathrm{XO}$ and even term series XP can be constructed:

$$
\begin{gathered}
\mathrm{XM}=1+\frac{1}{2}+\frac{1}{12}+\frac{1}{56}+\frac{1}{240}+\frac{1}{992}+\frac{1}{4032}+\frac{1}{16256}+\cdots=1+\sum_{i=1}^{\infty} \frac{1}{2^{i}\left(2^{i}-1\right)}=1.606695 \cdots ; \\
\mathrm{XN}=1-\frac{1}{2}+\frac{1}{12}-\frac{1}{56}+\frac{1}{240}-\frac{1}{992}+\frac{1}{4032}-\frac{1}{16256}+\cdots=1+\sum_{i=1}^{\infty}(-1)^{i} \frac{1}{2^{i}\left(2^{i}-1\right)}=0.568834 \cdots ; \\
\mathrm{XO}=1+\frac{1}{12}+\frac{1}{240}+\frac{1}{4032}+\frac{1}{65280}+\frac{1}{1047552}+\cdots=1+\sum_{i=1}^{\infty} \frac{1}{2^{2 i}\left(2^{2 i}-1\right)}=1.087764 \cdots ;
\end{gathered}
$$




$$
\mathrm{XP}=\frac{1}{2}+\frac{1}{56}+\frac{1}{992}+\frac{1}{16256}+\frac{1}{261632}+\cdots=\sum_{i=1}^{\infty} \frac{1}{2^{2 i-1}\left(2^{2 i-1}-1\right)}=0.518930 \cdots .
$$

The numerical value of the sum of series XM is equal to that of the sum of series XI, that is, the sum of the series XM also converges to the same value as the Erdös-Borwein constant (M. Chen, \& S. Chen, 2016). The Erdös-Borwein constant appears surprisingly twice in the half-life related series. Although the $i$ th term of series XM decreases more rapidly when $i \rightarrow \infty$ than the $i$ th term of series XI does, XM=XI still holds since the second term of series XM is larger than the second term of series XI. It is easy to attain: $\mathrm{XM}=\mathrm{XO}+\mathrm{XP}$ and $\mathrm{XN}=\mathrm{XO}-\mathrm{XP}$.

It is also found that one-half of the numerical value of the sum of series $\mathrm{XN}$ plus the sum of series $\mathrm{XJ}$ is equal to $2 / 3$, i.e., the sum of series $\mathrm{XB}$ or $\mathrm{XD}$, leading to the relations: $(\mathrm{XJ}+\mathrm{XN}) / 2=2 / 3=\mathrm{XB}=\mathrm{XD}, \mathrm{XJ}+\mathrm{XN}=4 / 3=\mathrm{XC}$, and $3(\mathrm{XJ}+\mathrm{XN}) / 2=\mathrm{XB}+\mathrm{XC}=\mathrm{XA}$. So, we further obtain the following interesting relations among the above series (except the divergent series $\mathrm{XE}$ to $\mathrm{XH}): \quad(\mathrm{XK}-\mathrm{XL}+\mathrm{XO}-\mathrm{XP}) / 2=2 / 3=\mathrm{XB}=\mathrm{XD}, \quad \mathrm{XK}-\mathrm{XL}+\mathrm{XO}-\mathrm{XP}=4 / 3=\mathrm{XC}$, $3(\mathrm{XK}-\mathrm{XL}+\mathrm{XO}-\mathrm{XP}) / 2=\mathrm{XB}+\mathrm{XC}=\mathrm{XA}, \quad(\mathrm{XJ}+\mathrm{XO}-\mathrm{XP}) / 2=\mathrm{XB}=\mathrm{XD}, \quad \mathrm{XJ}+\mathrm{XO}-\mathrm{XP}=\mathrm{XC}, \quad 3(\mathrm{XJ}+\mathrm{XO}-\mathrm{XP}) / 2=\mathrm{XA}$, $(\mathrm{XK}-\mathrm{XL}+\mathrm{XN}) / 2=\mathrm{XB}=\mathrm{XD}, \mathrm{XK}-\mathrm{XL}+\mathrm{XN}=\mathrm{XC}$, and $3(\mathrm{XK}-\mathrm{XL}+\mathrm{XN}) / 2=\mathrm{XA}$, and so on.

From the denominators of series XK, XL, XM, XO and XP, we can construct the following 5 increasing sequences:

$$
\begin{aligned}
& 1,7,31,127,511,2047,8191, \cdots, 2^{2 i-1}-1, \cdots ; \\
& (1), 3,15,63,255,1023,4095, \cdots, 2^{2 i}-1, \cdots ; \\
& (1), 2,12,56,240,992,4032,16256, \cdots, 2^{i}\left(2^{i}-1\right), \cdots ; \\
& (1), 12,240,4032,65280,1047552,16773120, \cdots, 2^{2 i}\left(2^{2 i}-1\right), \cdots ; \\
& (1), 2,56,992,16256,261632,4192256, \cdots, 2^{2 i-1}\left(2^{2 i-1}-1\right), \cdots .
\end{aligned}
$$

The above sequences are neither arithmetic sequences nor geometric sequences. When $i$ increases, they grow rapidly, especially the latter two sequences. The limits of $(i+1)$ term/ $i$ th term when $i \rightarrow \infty$ for these sequences are $4,4,4,16$, and 16 , respectively.

\section{Mean Life Related (or $\boldsymbol{e}$-Related) Infinite Series}

Another parameter that is sometimes used to describe the decay of a radioactive nuclide is the mean life, $\tau$, defined as the average life expectancy of a radioactive atom (Faure, 1977):

$$
\tau=\frac{1}{P(0)} \int_{0}^{\infty} \lambda P(t) t d t=\lambda \int_{0}^{\infty} t e^{-\lambda t} d t=\frac{1}{\lambda}
$$

We also obtain $\tau=T_{1 / 2} / \ln 2$. Therefore Equations (1) and (2) can also be rewritten using mean life as follows:

$$
P(j)=P(0) e^{-\lambda \tau(j)},
$$

and

$$
D(j)=P(0)\left(1-e^{-\lambda \tau(j)}\right),
$$

where $j$ is the number of mean life of decay of the radioactive parent isotope, $P(j)$ and $D(j)$ are the values of atoms of the radioactive parent isotope and the radiogenic daughter isotope after $j$ th mean life's decay, respectively. Both the decay of $P(j)$ and the increase of $D(j)$ with $j$ are also shown in Figure 1 with the same scale on the abscissa when $P(0)=120$. It is also easy to see that $P(j)=D(j)=60$ when $j=\ln 2$.

Obviously a radioactive nuclide is reduced by a factor equal to $1 / e$ of its initial value during each mean life (Faure, 1977). Similarly, if the initial value of the radioactive parent isotope is $P(0)$, we can give the information on decrease in the radioactive parent isotope and increase in the radiogenic daughter isotope in each mean life's decay during a single-component decay, as listed in Table 2. Also listed in Table 2 are the similar ratios as those in Table 1. 
Table 2. Decrease in the radioactive parent isotope and increase in the radiogenic daughter isotope during each mean life in a single-component decay.

\begin{tabular}{cccccccc}
\hline $\mathrm{D} 1$ & $\mathrm{D} 2$ & $\mathrm{D} 3$ & $\mathrm{D} 4$ & $\mathrm{D} 5$ & $\mathrm{D} 6$ & $\mathrm{D} 7$ & $\mathrm{D} 8$ \\
\hline$j$ & $\tau(j)$ & $P(j)$ & $D(j)$ & $\frac{P(j)}{P(0)}$ & $\frac{D(j)}{P(0)}$ & $\frac{P(j)}{D(j)}$ & $\frac{P(j)}{P(0)} \cdot \frac{P(j)}{D(j)}$ \\
\hline 1 & $1 / \lambda$ & $P(0) / e$ & $P(0)[(e-1) / e]$ & $1 / e$ & $(e-1) / e$ & $1 /(e-1)$ & $1 /[e(e-1)]$ \\
2 & $2 / \lambda$ & $P(0) / e^{2}$ & $P(0)\left[\left(e^{2}-1\right) / e^{2}\right]$ & $1 / e^{2}$ & $\left(e^{2}-1\right) / e^{2}$ & $1 /\left(e^{2}-1\right)$ & $1 /\left[e^{2}\left(e^{2}-1\right)\right]$ \\
3 & $3 / \lambda$ & $P(0) / e^{3}$ & $P(0)\left[\left(e^{3}-1\right) / e^{3}\right]$ & $1 / e^{3}$ & $\left(e^{3}-1\right) / e^{3}$ & $1 /\left(e^{3}-1\right)$ & $1 /\left[e^{3}\left(e^{3}-1\right)\right]$ \\
4 & $4 / \lambda$ & $P(0) / e^{4}$ & $P(0)\left[\left(e^{4}-1\right) / e^{4}\right]$ & $1 / e^{4}$ & $\left(e^{4}-1\right) / e^{4}$ & $1 /\left(e^{4}-1\right)$ & $1 /\left[e^{4}\left(e^{4}-1\right)\right]$ \\
5 & $5 / \lambda$ & $P(0) / e^{5}$ & $P(0)\left[\left(e^{5}-1\right) / e^{5}\right]$ & $1 / e^{5}$ & $\left(e^{5}-1\right) / e^{5}$ & $1 /\left(e^{5}-1\right)$ & $1 /\left[e^{5}\left(e^{5}-1\right)\right]$ \\
6 & $6 / \lambda$ & $P(0) / e^{6}$ & $P(0)\left[\left(e^{6}-1\right) / e^{6}\right]$ & $1 / e^{6}$ & $\left(e^{6}-1\right) / e^{6}$ & $1 /\left(e^{6}-1\right)$ & $1 /\left[e^{6}\left(e^{6}-1\right)\right]$ \\
7 & $7 / \lambda$ & $P(0) / e^{7}$ & $P(0)\left[\left(e^{7}-1\right) / e^{7}\right]$ & $1 / e^{7}$ & $\left(e^{7}-1\right) / e^{7}$ & $1 /\left(e^{7}-1\right)$ & $1 /\left[e^{7}\left(e^{7}-1\right)\right]$ \\
8 & $8 / \lambda$ & $P(0) / e^{8}$ & $P(0)\left[\left(e^{8}-1\right) / e^{8}\right]$ & $1 / e^{8}$ & $\left(e^{8}-1\right) / e^{8}$ & $1 /\left(e^{8}-1\right)$ & $1 /\left[e^{8}\left(e^{8}-1\right)\right]$ \\
9 & $9 / \lambda$ & $P(0) / e^{9}$ & $P(0)\left[\left(e^{9}-1\right) / e^{9}\right]$ & $1 / e^{9}$ & $\left(e^{9}-1\right) / e^{9}$ & $1 /\left(e^{9}-1\right)$ & $1 /\left[e^{9}\left(e^{9}-1\right)\right]$ \\
10 & $10 / \lambda$ & $P(0) / e^{10}$ & $P(0)\left[\left(e^{10}-1\right) / e^{10}\right]$ & $1 / e^{10}$ & $\left(e^{10}-1\right) / e^{10}$ & $1 /\left(e^{10}-1\right)$ & $1 /\left[e^{10}\left(e^{10}-1\right)\right]$ \\
$\ldots$ & $\ldots$ & $\ldots$ & $\ldots$ & $\ldots$ & $\ldots$ & $\ldots$ & $\ldots$
\end{tabular}

When 1 is added into column D5 in Table 2, from the fractions we can construct the infinite geometric series, including the following positive term series ZA, alternating series ZB, odd term series ZC and even term series ZD, and obtain their sums:

$$
\begin{gathered}
\mathrm{ZA}=1+\frac{1}{e}+\frac{1}{e^{2}}+\frac{1}{e^{3}}+\frac{1}{e^{4}}+\frac{1}{e^{5}}+\frac{1}{e^{6}}+\frac{1}{e^{7}}+\frac{1}{e^{8}}+\cdots=\sum_{j=0}^{\infty} \frac{1}{e^{j}}=\frac{e}{e-1}=1.581976 \cdots \\
\mathrm{ZB}=1-\frac{1}{e}+\frac{1}{e^{2}}-\frac{1}{e^{3}}+\frac{1}{e^{4}}-\frac{1}{e^{5}}+\frac{1}{e^{6}}-\frac{1}{e^{7}}+\frac{1}{e^{8}}-\cdots=\sum_{j=0}^{\infty}(-1)^{j} \frac{1}{e^{j}}=\frac{e}{e+1}=0.731058 \cdots ; \\
\mathrm{ZC}=1+\frac{1}{e^{2}}+\frac{1}{e^{4}}+\frac{1}{e^{6}}+\frac{1}{e^{8}}+\frac{1}{e^{10}}+\cdots=\sum_{j=0}^{\infty} \frac{1}{e^{2 j}}=\frac{e^{2}}{e^{2}-1}=1.156517 \cdots ; \\
\mathrm{ZD}=\frac{1}{e}+\frac{1}{e^{3}}+\frac{1}{e^{5}}+\frac{1}{e^{7}}+\frac{1}{e^{9}}+\cdots=\sum_{j=1}^{\infty} \frac{1}{e^{2 j-1}}=\frac{1}{e^{\infty}} \sum_{j=0}^{\infty} \frac{1}{e^{2 j}}=\frac{e}{e^{2}-1}=0.425459 \cdots
\end{gathered}
$$

It is found that $\mathrm{ZA}=\mathrm{ZC}+\mathrm{ZD}, \quad \mathrm{ZB}=\mathrm{ZC}-\mathrm{ZD}, \quad \mathrm{ZC}=\mathrm{ZA} \cdot \mathrm{ZB}, \quad \mathrm{ZC}=(\mathrm{ZA}+\mathrm{ZB}) / 2, \quad \mathrm{ZD}=\mathrm{ZC} / e, \quad \mathrm{ZD}=(\mathrm{ZA} \cdot \mathrm{ZB}) / e \quad$ and $\mathrm{ZD}=(\mathrm{ZA}-\mathrm{ZB}) / 2$.

When 1 is added into column D6 in Table 2, the following positive term series ZE, alternating series ZF, odd term series $\mathrm{ZG}$ and even term series $\mathrm{ZH}$ can be constructed:

$$
\begin{gathered}
\mathrm{ZE}=1+\frac{e-1}{e}+\frac{e^{2}-1}{e^{2}}+\frac{e^{3}-1}{e^{3}}+\frac{e^{4}-1}{e^{4}}+\frac{e^{5}-1}{e^{5}}+\frac{e^{6}-1}{e^{6}}+\cdots=1+\sum_{j=1}^{\infty} \frac{e^{j}-1}{e^{j}}=\infty ; \\
\mathrm{ZF}=1-\frac{e-1}{e}+\frac{e^{2}-1}{e^{2}}-\frac{e^{3}-1}{e^{3}}+\frac{e^{4}-1}{e^{4}}-\frac{e^{5}-1}{e^{5}}+\cdots=1+\sum_{j=1}^{\infty}(-1)^{j} \frac{e^{j}-1}{e^{j}}=\left\{\begin{array}{c}
1.268941 \cdots \\
0.268941 \cdots
\end{array}\right. \\
\mathrm{ZG}=1+\frac{e^{2}-1}{e^{2}}+\frac{e^{4}-1}{e^{4}}+\frac{e^{6}-1}{e^{6}}+\frac{e^{8}-1}{e^{8}}+\frac{e^{10}-1}{e^{10}}+\cdots=1+\sum_{j=1}^{\infty} \frac{e^{2 j}-1}{e^{2 j}}=\infty ; \\
\mathrm{ZH}=\frac{e^{2 j}}{e}+\frac{e^{3}-1}{e^{3}}+\frac{e^{5}-1}{e^{5}}+\frac{e^{7}-1}{e^{7}}+\frac{e^{9}-1}{e^{9}}+\cdots=\sum_{j=1}^{\infty} \frac{e^{2 j-1}-1}{e^{2 j-1}}=\infty
\end{gathered}
$$

Series ZE, ZG and ZH are obviously divergent. Series ZF also diverges since when $j \rightarrow \infty$, its $j$ th term is not equal to 0 and the partial sum approaches alternatively to the upper bounded value $\mathrm{ZFU}=1.268941 \cdots$ and the lower bounded value $\mathrm{ZFL}=0.268941 \cdots$ (Figure 2 ). It is easy to obtain: $\mathrm{ZFU}-\mathrm{ZFL}=1$. 


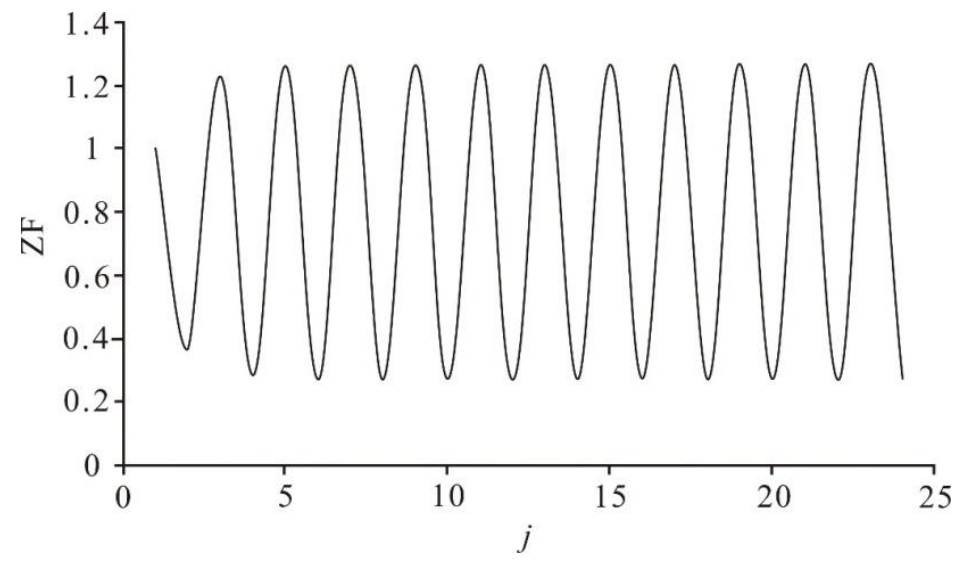

Figure 2. Change in the partial sum of series ZF with $j(1 \leq j \leq 24)$

From column D7 in Table 2, i.e., the ratios of the remaining numbers of the radioactive parent isotope to the accumulating numbers of the radiogenic daughter isotope for each mean life's decay, we can write the following positive term series ZI, alternating series ZJ, odd term series ZK and even term series ZL:

$$
\begin{gathered}
\mathrm{ZI}=\frac{1}{e-1}+\frac{1}{e^{2}-1}+\frac{1}{e^{3}-1}+\frac{1}{e^{4}-1}+\frac{1}{e^{5}-1}+\frac{1}{e^{6}-1}+\frac{1}{e^{7}-1}+\cdots=\sum_{j=1}^{\infty} \frac{1}{e^{j}-1}=0.820259 \cdots ; \\
\mathrm{ZJ}=\frac{1}{e-1}-\frac{1}{e^{2}-1}+\frac{1}{e^{3}-1}-\frac{1}{e^{4}-1}+\frac{1}{e^{5}-1}-\frac{1}{e^{6}-1}+\frac{1}{e^{7}-1}-\cdots=\sum_{j=1}^{\infty}(-1)^{j+1} \frac{1}{e^{j}-1}=0.464163 \cdots ; \\
\mathrm{ZK}=\frac{1}{e-1}+\frac{1}{e^{3}-1}+\frac{1}{e^{5}-1}+\frac{1}{e^{7}-1}+\frac{1}{e^{9}-1}+\cdots=\sum_{j=1}^{\infty} \frac{1}{e^{2 j-1}-1}=0.642211 \cdots ; \\
\mathrm{ZL}=\frac{1}{e^{2}-1}+\frac{1}{e^{4}-1}+\frac{1}{e^{6}-1}+\frac{1}{e^{8}-1}+\frac{1}{e^{10}-1}+\cdots=\sum_{j=1}^{\infty} \frac{1}{e^{2 j}-1}=0.178047 \cdots ;
\end{gathered}
$$

It is found that $\mathrm{ZI}=\mathrm{ZK}+\mathrm{ZL}$ and $\mathrm{ZJ}=\mathrm{ZK}-\mathrm{ZL}$.

From column D8 in Table 2, the following positive term series ZM, alternating series ZN, odd term series ZO and even term series ZP can be constructed:

$$
\begin{gathered}
\mathrm{ZM}=\frac{1}{e(e-1)}+\frac{1}{e^{2}\left(e^{2}-1\right)}+\frac{1}{e^{3}\left(e^{3}-1\right)}+\frac{1}{e^{4}\left(e^{4}-1\right)}+\frac{1}{e^{5}\left(e^{5}-1\right)}+\cdots=\sum_{j=1}^{\infty} \frac{1}{e^{j}\left(e^{j}-1\right)}=0.238282 \cdots ; \\
\mathrm{ZN}=\frac{1}{e(e-1)}-\frac{1}{e^{2}\left(e^{2}-1\right)}+\frac{1}{e^{3}\left(e^{3}-1\right)}-\frac{1}{e^{4}\left(e^{4}-1\right)}+\cdots=\sum_{j=1}^{\infty}(-1)^{j+1} \frac{1}{e^{j}\left(e^{j}-1\right)}=0.195222 \cdots ; \\
\mathrm{ZO}=\frac{1}{e(e-1)}+\frac{1}{e^{3}\left(e^{3}-1\right)}+\frac{1}{e^{5}\left(e^{5}-1\right)}+\frac{1}{e^{7}\left(e^{7}-1\right)}+\cdots=\sum_{j=1}^{\infty} \frac{1}{e^{2 j-1}\left(e^{2 j-1}-1\right)}=0.216752 \cdots ; \\
\mathrm{ZP}=\frac{1}{e^{2}\left(e^{2}-1\right)}+\frac{1}{e^{4}\left(e^{4}-1\right)}+\frac{1}{e^{6}\left(e^{6}-1\right)}+\frac{1}{e^{8}\left(e^{8}-1\right)}+\cdots=\sum_{j=1}^{\infty} \frac{1}{e^{2 j}\left(e^{2 j}-1\right)}=0.021530 \cdots .
\end{gathered}
$$

It is also found that $\mathrm{ZM}=\mathrm{ZO}+\mathrm{ZP}$ and $\mathrm{ZN}=\mathrm{ZO}-\mathrm{ZP}$.

\section{Summary}

Sixteen half-life related infinite series and 16 mean life related infinite series can be constructed by examining the changes in atoms of the radioactive parent isotope and the radiogenic daughter isotope for each half-life's and mean life's decay, respectively. Four half-life related series (XE, XF, XG and XH) and 4 mean life related series (ZE, ZF, ZG and $\mathrm{ZH})$ are divergent. Four convergent half-life related series $(\mathrm{XA}, \mathrm{XB}, \mathrm{XC}$ and $\mathrm{XD})$ are previously known, and 2 half-life related series (XI and XM) converge to the Erdös-Borwein constant. From the remaining convergent series, 18 mathematical constants (XJ, XK, XL, XN, XO, XP, ZA, ZB, ZC, ZD, ZI, ZJ, ZK, ZL, ZM, ZN, ZO and ZP) are acquired. From the upper and lower bounded values of the divergent and alternating mean life related series $Z F, 2$ more mathematical constants (ZFU and ZFL) are obtained. Five sequences are also constructed from the convergent half-life related series (XK, XL, XM, XO and XP). It is also found that there is a close link and exist a few interesting mathematical relations among the sums of the convergent series. The half-life related and mean life related series are 2-related and $e$-related, respectively. 


\section{Acknowledgements}

This work was supported by the National Natural Science Foundation of China (41772261).

\section{References}

Alzer, H., Karayannakis, D., \& Srivastava, H. M. (2006). Series representations for some mathematical constants. Journal of Mathematical Analysis and Applications, 320, 145-162. https://doi.org/10.1016/j.jmaa.2005.06.059

Borwein, P. B. (1992). On the irrationality of certain series. Mathematical Proceedings of the Cambridge Philosophical Society, 112, 141-146. https://doi.org/10.1017/S030500410007081X

Chen, M., \& Chen, S. (2016). Interesting Mathematical Constants. Beijing: Posts \& Telecom Press. (in Chinese).

Chen, R. (2011). The Code of e. Beijing: Science Press. (in Chinese)

Choi, J. (2012). A set of mathematical constants arising naturally in the theory of the multiple Gamma functions. Abstract and Applied Analysis, Article ID 121795. https://doi.org/10.1155/2012/121795

Erdös, P. (1948). On the arithmetical properties of Lambert series. Journal of the Indian Mathematical Society (N. S.), 12, 63-66.

Faure, G. (1977). Principles of Isotope Geology. New York: John Wiley and Sons.

Finch, S. R. (2003). Mathematical Constants. Cambridge: Cambridge University Press.

Lutgens, F. K., \& Tarbuck, E. J. (2014). Foundations of Earth Science (7th ed.). New York: Pearson Education, Inc.

Turcotte, D. L., \& Schubert, G. (2002). Geodynamics (2nd ed.). Cambridge: Cambridge University Press. https://doi.org/10.1017/CBO9780511807442

\section{Copyrights}

Copyright for this article is retained by the author(s), with first publication rights granted to the journal.

This is an open-access article distributed under the terms and conditions of the Creative Commons Attribution license (http://creativecommons.org/licenses/by/4.0/). 